phase 2a dose escalation study to investigate the safety, PK, PD, and efficacy of CC-220 in patients with SLE. Forty-two (42) adult SLE subjects fulfilling SLE ACR criteria, having a history of SLE for $\geq 6$ months and a baseline Safety of Estrogens in Lupus Erythematosus National Assessment-Systemic Lupus Erythematosus Disease Activity Index (SELENA-SLEDAI) score $\geq 4$ were randomized to placebo or 1 of 4 escalating doses of CC-220 $(0.3 \mathrm{mg} \mathrm{QOD}, 0.3 \mathrm{mg} \mathrm{QD}, 0.6 / 0.3 \mathrm{mg}$ alternating $\mathrm{QD}$, or $0.6 \mathrm{mg} \mathrm{QD)}$.

Results: CC-220 concentration-time profiles demonstrated dose proportionality between cohorts, with moderate accumulation in the non-alternating dose cohort. CC-220 significantly reduced total CD20+ B cells by as much as $96 \%$, immature B cells by as much as $91.2 \%$, switched memory B cells by as much as $81.4 \%$, BAFFR+ B cells by as much as $67.5 \%$, and plasmacytoid dendritic cells (pDCs) by as much as $86.5 \%$ (Day 85 median percent change from baseline). Whereas reductions in B cells were observed, CD4+ as well as CD8+ T cells increased, and the rise in $T$ cells paralleled the observed increase in plasma cells in those subjects who received the highest dose $(0.6 \mathrm{mg})$. An exposure-response analysis demonstrated decreasing $B$ cells, pDCs and neutrophils with increased exposure to CC-220.

Conclusions: It was determined that $0.3 \mathrm{mg} \mathrm{QD}$ to $0.6 / 0.3 \mathrm{mg}$ alternating $\mathrm{QD}$ reduced concentrations of $\mathrm{B}$ cells and pDC's while avoiding neutropenia. These findings, in combination with the PK, safety, and exploratory efficacy data, support continued development of CC-220 in SLE.

Disclosure of Interest: A. Gaudy Shareholder of: Celgene, Employee of: Celgene, Y. Ye Shareholder of: Celgene, Employee of: Celgene, S. Korish Shareholder of: Celgene, Employee of: Celgene, D. Hough Shareholder of: Celgene, Employee of: Celgene, M. Weiswasser Shareholder of: Celgene, Employee of: Celgene, S. Choi Shareholder of: Celgene, Employee of: Celgene, R. Furie Consultant for: Celgene, V. Werth Grant/research support from: Celgene, Consultant for: Celgene, P. Schafer Shareholder of: Celgene, Employee of: Celgene

DOI: 10.1136/annrheumdis-2017-eular.3036

\section{SAT0226 A FIRST-IN-HUMAN, STUDY OF BMS-986165, A SELECTIVE, POTENT, ALLOSTERIC SMALL MOLECULE INHIBITOR OF TYROSINE KINASE 2}

I. Catlett ${ }^{1}$, U. Aras $^{2}$, Y. Liu ${ }^{3}$, D. Bei ${ }^{2}$, I. Girgis ${ }^{2}$, B. Murthy ${ }^{2}$, M. Honczarenko ${ }^{1}$ S. Rose ${ }^{1} .{ }^{1}$ Innovative Clinical Development; ${ }^{2}$ Clinical Pharmacology; ${ }^{3}$ Global Biometric Sciences, Bristol-Myers Squibb, Princeton, United States

Background: Tyrosine kinase 2 (Tyk2) is a member of the JAK family that phosphorylates STAT proteins downstream of the IL-12, IL-23 and the Type I interferon receptor. Tyk2 genetic variants have been linked to multiple autoimmune diseases, ${ }^{1}$ with a deactivating coding variant conferring significant protection against multiple immune-mediated disorders including systemic lupus erythematosus (SLE). ${ }^{2}$ Selective pharmacologic inhibition of Tyk2 has proven daunting, given the high degree of similarity among JAK catalytic domains. Using a novel approach, we have developed BMS-986165, a highly selective and potent small molecule inhibitor, which blocks receptor-mediated Tyk2 activation by stabilizing the regulatory pseudokinase domain of the protein. Lupus-like disease is strongly inhibited in NZB/W mice treated with BMS-986165. Together, these data establish Tyk2 as a highly promising therapeutic target for SLE.

Objectives: We report the first evidence of safety, pharmacokinetics (PK), target engagement (TE), and pharmacodynamic activity (PD) of BMS-986165, a novel inhibitor of Tyk2.

Methods: Safety, PK, TE, and PD were assessed in a randomized, double-blind, single and multiple ascending dose study of 108 ( 83 active: 25 placebo) healthy participants (NCT02534636). Target engagement was assessed by an ex vivo assay, IL-12 and IL-18 induced IFN $\gamma$ production. Roferon-A was administered to assess in vivo pharmacodynamic effects of BMS-986165 on physiological manifestations of IFN exposure and on IFN-regulated gene (IRG) expression.

Results: BMS-986165 was safe and overall well-tolerated. There were no serious adverse events and the frequency of non-serious adverse events were similar in the active $(75 \%)$ and placebo $(76 \%)$ groups. The most frequently reported adverse events by preferred term were headache $(23 \%$ active versus $28 \%$ placebo), nausea (12\% active versus $8 \%$ placebo), rash (12\% active versus $8 \%$ placebo), and upper respiratory tract infection ( $11 \%$ active versus $12 \%$ placebo). After oral administration, BMS-986165 was rapidly absorbed and exhibited an apparent elimination half-life of $8-15$ hours. Modest accumulation $(1.4 x-1.9 x)$ was observed after multiple dosing. BMS-986165 inhibited IL-12/IL-18-induced IFN $\gamma$ production in ex vivo assays in a dose and concentration dependent manner. Following an in vivo challenge with a clinical dose of Interferon-alfa-2A, BMS986165 demonstrated dose-dependent inhibition of lymphocyte count decreases and expression of 53 IRGs in blood (Figure 1).

Conclusions: BMS-986165 is a safe and potent Tyk2 inhibitor with clear evidence of ex vivo and in vivo biologic activity in healthy participants, and the potential for once daily dosing. Overall, Inhibition of IL-12/23 and type I IFN pathways support further testing of BMS-986165 in diseases such as lupus and psoriasis. A Phase 2 study in patients with moderate-to-severe psoriasis is ongoing (NCT02931838). References:

[1] Liang Y, Zhu Y, Xia Y, Peng H, Yang XK, et al. Therapeutic potential of tyrosine kinase 2 in autoimmunity. Expert Opin Ther Targets. 2014 May;18(5):57180.

[2] Dendrou CA, Cortes A, Shipman L, Evans HG, Attfield KE, et al. Resolving
Figure 1: In vivo pharmacodynamic challenge
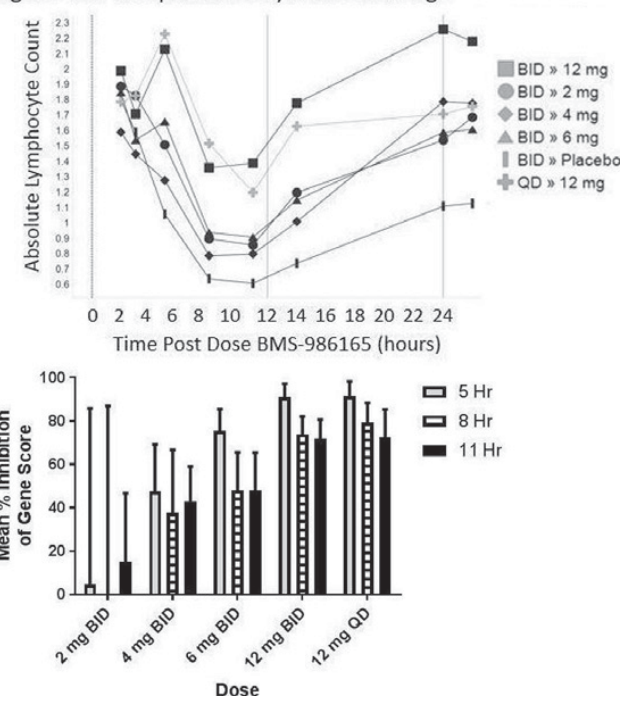

TYK2 locus genotype-to-phenotype differences in autoimmunity. Sci Transl Med. 2016 Nov 2;8(363):363ra149.

Disclosure of Interest: I. Catlett Shareholder of: Bristol-Myers Squibb, Employee of: Bristol-Myers Squibb, U. Aras Shareholder of: Bristol-Myers Squibb, Employee of: Bristol-Myers Squibb, Y. Liu Shareholder of: Bristol-Myers Squibb, Employee of: Bristol-Myers Squibb, D. Bei Shareholder of: Bristol-Myers Squibb, Employee of: Bristol-Myers Squibb, I. Girgis Shareholder of: Bristol-Myers Squibb, Employee of: Bristol-Myers Squibb, B. Murthy Shareholder of: Bristol-Myers Squibb, Employee of: Bristol-Myers Squibb, M. Honczarenko Shareholder of: Bristol-Myers Squibb, Employee of: Bristol-Myers Squibb, S. Rose Shareholder of: Bristol-Myers Squibb, Employee of: Bristol-Myers Squibb

DOI: 10.1136/annrheumdis-2017-eular.3809

\section{SAT0227 HEALTH CARE UTILIZATION AND COSTS OF SYSTEMIC LUPUS ERYTHEMATOSUS (SLE) IN THE UNITED STATES: SYSTEMATIC REVIEW}

E. Hammond ${ }^{1}$, I.B. Murimi ${ }^{2}$, D.H. Lin ${ }^{2}$, H. Kan ${ }^{2}$, J. Tierce ${ }^{2}$, X. Wang ${ }^{1}$, H. Nab ${ }^{3}$, B. Desta ${ }^{1}$, G.C. Alexander ${ }^{2} .{ }^{1}$ AstraZeneca, Gaithersburg, MD; ${ }^{2}$ Johns Hopkins Bloomberg School of Public Health, Baltimore, MD, United States; ${ }^{3}$ AstraZeneca, Cambridge, United Kingdom

Background: Systemic lupus erythematosus (SLE) is a chronic autoimmune disease that affects as many as 518 persons per 100,000 people in the United States and Europe.

However, much of our current knowledge on SLE health care utilization and costs is based on analyses conducted prior to 2011, after which important treatment innovations (e.g., belimumab and, to some extent, rituximab) and health care marketplace changes occurred in the United States.

Objectives: The objectives of this systematic review were to summarize the patterns of health care utilization for patients with SLE and to characterize the direct and indirect costs of SLE by disease severity in the United States.

Methods: We conducted a systematic literature review of adult patients with SLE in the United States by searching PubMed and Embase for relevant articles written in English and published during January 2000-April 2016. We examined health care utilization measures including emergency department (ED) visits, hospitalizations, outpatient visits, medication use, and adherence. We restricted the analysis to observational studies, excluding case reports and commentaries. All monetary costs were converted to US 2016 \$.

Results: In total, 4,700 articles were screened, 388 articles were retained for full-text review, and 38 articles were selected for inclusion in this report. Across these 38 studies, mean patient ED utilization rates were 0.9-2.1 visits/year. Mean hospitalization rates were 0.4-2.6 inpatient stays/year, with 5-6 days/stay on average. Patients averaged 10-19 total physician visits/year. More than $90 \%$ of patients with SLE had $\geq 1$ visit to a primary care provider annually. In addition, $51-71 \%$ visited rheumatologists, and $6-7 \%$ visited nephrologists. Health care resource use with respect to ED visits, hospitalization rates, inpatient length of stay, and ambulatory care was greater for patients with lupus nephritis than for patients with SLE without nephritis. Mean annual direct costs from various studies ranged between $\$ 15,171-88,445$ for patients with SLE regardless of the presence of nephritis. Nearly all studies indicated that mean direct medical costs were greater for patients with moderate or severe disease $(\$ 22,300-83,000)$ than those with mild disease $(\$ 8,900-15,000)$. Mean annual pharmacy costs ranged between $\$ 1,572-13,138$, accounting for $19-23 \%$ of total direct costs, and medical costs ranged between $\$ 21,290-82,854$. On average, $12-49 \%$ of patients were unemployed, and the mean number of sick days/month was 2.3 days. The range of estimates of mean total direct costs for patients with SLE overall were often 
larger when derived from commercial claims $(\$ 21,600-55,400)$ than from public payers (Medicare and Medicaid, \$16,000-23,000).

Conclusions: Our findings suggest that patients with SLE, especially those with moderate or severe disease, use considerably more health care services and incur greater direct and indirect costs relative to those with mild disease. Thus, SLE remains a significant driver of health care resource utilization and costs.

Disclosure of Interest: E. Hammond Employee of: AstraZeneca, I. Murimi: None declared, D. Lin: None declared, H. Kan Shareholder of: GSK, J. Tierce: None declared, X. Wang Employee of: AstraZeneca, H. Nab Employee of: AstraZeneca, B. Desta Employee of: AstraZeneca, G. C. Alexander: None declared DOI: 10.1136/annrheumdis-2017-eular.5224

\section{SAT0228 APOPTOTIC EFFECT OF BLYS ON ENDOTHELIAL CELLS AND ENDOTHELIAL PROGENITOR CELLS IS MEDIATED BY BLYS RECEPTROS AND IS REVERTED BY BELIMUMAB}

F.R. Spinelli, C. Barbati, F. Ceccarelli, T. Colasanti, F. Morello, L. Massaro, V. Orefice, C. Alessandri, F. Conti, G. Valesini. Dipartimento di Medicina Interna e Specialità Mediche - Reumatologia, Sapienza Università di Roma, Rome, Italy

Background: Circulating endothelial progenitor cells (EPCs) are surrogate markers of endothelial function. Several studies demonstrated a reduction and functional impairment of EPCs in patients with Systemic Lupus Erythematosus (SLE), partially accounting for endothelial dysfunction. In murine models of atherosclerosis, treatment with a B Lymphocyte Stimulator (BLyS) inhibitor slowed the progression and reduced the size of atherosclerotic plaque. Belimumab (BLM) is a human anti-BLyS monoclonal antibody approved for the treatment of SLE. Objectives: We aimed at evaluating the effect of BLyS inhibition on EPCs and endothelial cells both ex vivo - in SLE patients receiving BLM- and in vitro. Moreover we investigated the expression of receptors for BLyS on EPC and mature endothelial cell surface.

Methods: We enrolled consecutive patients with SLE who were due to start BLM, without known cardiovascular disease and age and sex-matched healthy subjects. Peripheral blood mononuclear cells (PBMC) were isolated by Ficoll densitygradient centrifugation. Cells were incubated with anti-CD34 and anti-VEGFR2/KDR monoclonal antibodies; acquisition was performed by flow cytometry: EPCs were defined as CD34/KDR double-positive cells. Recovered EPC isolated from healthy donors' PBMC were plated on dishes coated with human fibronectin. Apoptosis was investigated after 6, 12 and 24 hours of incubation with BLyS at different concentrations - 5, 20 and $100 \mathrm{ng} / \mathrm{ml}$ - and re-evaluated after 6 hours of co-incubation with BLM at 173 and $300 \mu \mathrm{g} / \mathrm{ml}$. The same experiments were repeated with the human endothelial cell line EA.hy926. Finally, EPCs and EA.hy926 were incubated with monoclonal antibodies anti-B Activating FactorReceptor (BAFF-R), B-cell maturation antigen (BCMA) and transmembrane activator and calcium modulator and cyclophilin ligand (CAML) interactor (TACI) and analysed by flow cytometry; the results were expressed as mean fluorescence intensity (MFI).

Results: We treated with BLM 10 female patients (mean age $45.6 \pm 10.2 \mathrm{yrs}$, mean disease duration $17.8 \pm 10.8 \mathrm{yrs}$ ) with active disease (mean baseline SLEDAI 8.4 2.6). Number of EPCs was significantly lower in SLE patients than in NHS $(p=0.005)$. After 4 weeks of BLM, mean EPC number increased from $0.013 \pm 0.016$ to $0.021 \pm 0.016$ ( $p=0.012$ vs baseline; $p=n$.s. vs NHS). At week 12 , EPC number did not significantly differ compared to week 4 nor to baseline.

In vitro studies demonstrated that $20 \mathrm{ng} / \mathrm{ml}$ of BLyS induced apoptosis of EPC after 6 hours of incubation; this effect was reverted by the addiction of BLM. Similarly, after 6 hours of incubation with $20 \mathrm{ng} / \mathrm{ml}$ of BLyS we detected an increase in EA.hy926 apoptosis that was reverted by co-incubation BLM. Both EPCs and EA.hy926 expressed on their surface BAFF-R (MFI $=3.8$ and 1.5, respectively) and $\mathrm{BCMA}(\mathrm{MFI}=1.25$ and 1.15, respectively); EPCs also expressed $\mathrm{TACl}(\mathrm{MFI}=1.4)$.

Conclusions: The results of this study demonstrated that the reduction of EPCs number detected in SLE patients was restored by BLM. In vitro results support a direct pro-apoptotic effect of BLyS that was reverted by the addition of BLM both in EPCs and EA.hy926 culture. The apoptotic effect of BLyS seems to be mediated by the three receptors (BAFF-R, BCMA and TACI) that are expressed on EPCs and mature endothelial cells surface.

Disclosure of Interest: None declared

DOI: 10.1136/annrheumdis-2017-eular.6174

\section{SAT0229 THE USE OF ANTIMALARIAL DRUGS DURING PREGNANCY CAN PREVENT THE DEVELOPMENT OF PREECLAMPSIA IN WOMEN WITH SYSTEMIC LUPUS ERYTHEMATOSUS}

M.A. Saavedra ${ }^{1}$, D. Miranda-Hernández ${ }^{1}$, A. Lara-Mejía ${ }^{1}$, A. Sánchez ${ }^{1}$, C.V. Cruz-Reyes ${ }^{1}$, U. Angeles ${ }^{2}$, L.J. Jara ${ }^{3}{ }^{1}{ }^{1}$ Rheumatology; ${ }^{2}$ Epidemiology Direction; ${ }^{3}$ Education and Research Direction, Hospital de Especialidades, Cmn la Raza, IMSS, México, Mexico

Background: The antimalarial drugs decrease the risk of lupus activity during gestation, but the beneficial effect on other maternal-fetal complications is controversial.

Objectives: To analyze the beneficial effect of antimalarial drugs on maternal-fetal complications in pregnant women with systemic lupus erythematosus (SLE).
Methods: A prospective cohort of pregnant women with SLE (ACR 1997) from January 2009 to June 2015 was studied. The patients were assessed every 4 to 6 weeks and postpartum both, by a rheumatologist and a gynecologist. Clinical, biochemical, and immunological characteristics, along with maternal and fetal complications were registered. For analysis, the patients were allocated to one of two groups: pregnancies exposed to antimalarial drugs in comparison to those not exposed. A logistic regression analysis including variables such as smoking, obesity, infections, first pregnancy, age, SLE flare, drugs (prednisone, antimalarials, aspirin, and azathioprine), anti-DNA antibodies, anticardiolipin antibodies, and antiphospholipid syndrome was performed.

Results: We studied 197 lupus pregnancies, 154 expose to antimalarial drugs and 47 unexposed. We found no differences between groups in age, years of evolution of SLE, first pregnancy, childhood-onset SLE, lupus nephritis, and use of prednisone, aspirin and azathioprine. The rate of most maternal and fetal complications was also similar in both groups (Table). A lower incidence of preeclampsia was observed in patients exposed to antimalarial drugs compared to those not exposed $(9 \%$ vs $23 \%, p=0.01)$. Additionally, 2 maternal deaths in patients not exposed to antimalarial drugs. The logistic regression analysis showed that the use of antimalarial drugs during pregnancy is a protective factor for the development of preeclampsia (RR $0.1,95 \% \mathrm{Cl} 0.05-0.58, \mathrm{p}=0.004$ ); on the other hand, active SLE before pregnancy (RR $4.8,95 \% \mathrm{Cl} 1.3-17.8, \mathrm{p}=0.01$ ) and lupus nephritis (RR $2.9,95 \% \mathrm{Cl} 0.9-8.8, \mathrm{p}=0.05$ ) were associated factors with the development of preeclampsia.

Table 1. Maternal and fetal outcomes

\begin{tabular}{lccc} 
& Antimalarial drugs $(\mathrm{n}=154)$ & No antimalarial drugs $(\mathrm{n}=43)$ & $\mathrm{P}$ value \\
\hline Maternal complications & $74(48.0)$ & $18(41.8)$ & 0.47 \\
Preeclampsia & $14(9.0)$ & $10(23.2)$ & 0.01 \\
PROM & $15(9.7)$ & $1(2.3)$ & 0.12 \\
Cesarean section & $99(64.2)$ & $29(67.4)$ & 0.53 \\
Maternal death & $0(0)$ & $2(4.6)$ & 0.007 \\
SLE flare & $60(38.9)$ & $8(18.6)$ & 0.01 \\
Renal flare & $23(14.9)$ & $5(11.6)$ & 0.58 \\
Infection & $21(13.6)$ & $5(11.6)$ & 0.73 \\
Fetal complications a & $71(46.1)$ & $22(51.1)$ & $0.56 \mathrm{~b}$ \\
Live births a & $137(88.9)$ & $38(88.3)$ & $0.98 \mathrm{~b}$ \\
Prematurity a & $40(25.9)$ & $13(30.2)$ & $0.58 \mathrm{~b}$ \\
Miscarriage a & $13(8.4)$ & $5(11.6)$ & $0.52 \mathrm{~b}$ \\
Stillbirth a & $4(2.5)$ & $0(0)$ & $0.28 \mathrm{~b}$ \\
Weeks' gestation c & $35.1 \pm 5.9$ & $35.0 \pm 6.3$ & $0.89 \mathrm{~d}$ \\
Birth weight (g) c & $2,534 \pm 651$ & $2,444 \pm 827$ & $0.5 \mathrm{~d}$ \\
Apgar score 1 c & $7.4 \pm 1.6$ & $7.2 \pm 1.6$ & $0.41 \mathrm{~d}$ \\
Apgar score 2 c & $8.6 \pm 1.4$ & $8.3 \pm 1.3$ & $0.37 \mathrm{~d}$ \\
\hline a Results expressed in mean $(\mathrm{SD})$ & b Student's t-test. ${ }^{c}$ Results expressed in numbers and percent
\end{tabular}

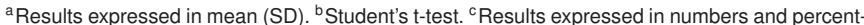
ages. ${ }^{\mathrm{d}}$ Chi-square test.

Conclusions: Our study suggests that the use of antimalarial drugs during pregnancy can prevent the development of preeclampsia in women with SLE.

Disclosure of Interest: None declared

DOI: 10.1136/annrheumdis-2017-eular.6035

\section{SAT0230 NEW ORAL ANICOAGULANTS IN PATIENTS WITH ANTIPHOSPHOLIPID SYNDROME}

M.A. Satybaldyeva, N. Seredavkina, L. Kashnikova, E. Nasonov, T. Reshetnyak. Nasonova Research Institute of Rheumatology, MOSCOW, RUSSIA, Moscow, Russian Federation

Background: Antiphospholipid syndrome (APS) is an acquired thrombophilia characterized by reccurent venous and arterial thrombosis, obstetric pathology (fetal loss), and synthesis of antiphospholipid antibodies. Warfarin is a "golden" standard of APS therapy. However it has number of disadvantages. Dabigatran etexilate is a direct thrombin inhibitor and its main differences from warfarin are fixed dose, no need of regular INR monitoring,less elimination half-life.

Objectives: To evaluate efficacy and safety of dabigatran etexilate in patients with APS.

Methods: 38 patients (pts) (F:26, M:12) with primary and secondary APS, $37,2 \pm 9,9$ years old. 24 pts with primary APS, 14 pts with secondary APS: 13 had systemic lupus erythematosus (SLE) + APS, 1 rheumathoid arthritis (RA) + APS. The diagnosis of APS was established due to international APS criteria (Sydney), SLE - SLICC 2012, RA - ACR/EULAR 2010. The majority number of pts $(n=28)$ received warfarin, others - sulodexide $(n=1)$, low molecular heparin $(n=1)$, had no anticoagulant therapy $(n=3), 5$ pts received dabigatran etexilate before inclusion to trial. The control of coagulogram was done 3 times: before inclusion to trial, in 24 weeks and in 48 weeks after inclusion. Trial assays were performed in the laboratory in V.A. Nasonova Research Institute of Rheumatology, Moscow, Russian Federation. APPT and thrombin time tests were done with the automated coagulometer Coalysys Plus C (Behnk Electronic, Germany); thrombin time test was done with STA-thrombin reagent (Diagnostica Stago, France), APPT with STA-Cephascreen reagent (Diagnostica Stago, France). Lupus anticoagulant was assessed by the dilute Russell's viper venom time, using Siemens Healthcare (Germany) LA1 (screening) and LA2 (confirmation). IgG or IgM antibodies against cardiolipin and $\beta 2$ glycoprotein I ( $\beta 2 \mathrm{GPI}$ ) were measured with automated enzyme-immunoassay analyzer Alegria with Anti-Cardiolipin IgG/lgM and Antibeta-2-Glycoprotein I lgG/lgM reagents (Orgentec Diagnostika GmbH, Germany). 